\title{
Xanthomonas hortorum pv. gardneri TAL effector AvrHah1 is necessary and sufficient for increased persistence of Salmonella enterica on tomato leaves
}

\author{
Kimberly N. Cowles \\ University of Wisconsin-Madison \\ Anna K. Block \\ United States Department of Agriculture \\ Jeri D. Barak ( $\sim$ jeri.barak@wisc.edu ) \\ University of Wisconsin-Madison
}

\section{Research Article}

Keywords: X. gardneri, biomultiplier, TAL effector, plant immunity, pathogen triggered immunity

Posted Date: January 20th, 2022

DOI: https://doi.org/10.21203/rs.3.rs-1229600/v1

License: (c) (i) This work is licensed under a Creative Commons Attribution 4.0 International License.

Read Full License 


\section{Abstract}

Salmonella enterica is ubiquitous in the plant environment, persisting in the face of UV stress, plant defense responses, desiccation, and nutrient limitation. These fluctuating conditions of the leaf surface result in $S$. enterica population decline. Biomultipliers, such as the phytopathogenic bacterium Xanthomonas hortorum pv. gardneri, alter the phyllosphere to the benefit of $S$. enterica. Specific $X$. gardneri-dependent changes to this niche that promote $S$. enterica persistence remain unclear, and this work focuses on identifying factors that lead to increased $S$. enterica survival on leaves. Here, we show that the $X$. gardneri transcription activator-like effector AvrHah1 is both necessary and sufficient for increased survival of $S$. enterica on tomato leaves. An $X$. gardneri avrHah1 mutant fails to influence $S$. enterica survival while addition of avrHah 1 to $X$. vesicatoria provides a gain of function. Our results indicate that although $X$. gardneri stimulates a robust immune response from the plant, AvrHah1 is not required for these effects. In addition, we demonstrate that cellular leakage that occurs during disease is independent of AvrHah1. Investigation of the interaction between S. enterica, X. gardneri, and the plant host provides information regarding how an inhospitable environment changes during infection and can be transformed into a habitable niche.

\section{Introduction}

Salmonella enterica is a human enteric pathogen that causes disease in approximately 1.2 million Americans annually (CDC). Numerous multi-state outbreaks of salmonellosis have occurred from the consumption of fresh produce every year since 2000 (National Outbreak Reporting System (NORS; ${ }^{1-4}$ )). $S$. enterica survives on multiple agricultural crops after root or leaf immigration ${ }^{5-7}$, and the bacteria persist in soil for months $s^{6,8,9}$. Despite the continued isolation of $S$. enterica from the agricultural environments associated with outbreaks, $S$. enterica has relatively poor fitness in the plant environment. Bacterial populations steadily decline in the phyllosphere, or above-ground parts of plants ${ }^{8,9}$.

The rapidly fluctuating conditions on the leaf surface provide a harsh environment for epiphytic bacteria, and survival depends on the ability to adapt to abrupt changes in UV irradiation and the availability of water and nutrients. Upon arrival to the leaf surface, S. enterica migrates to protected sites like trichomes, stomates, hydathodes, and epidermal cell wall junctions $s^{7,10-12}$. These locations are often associated with a local increase in nutrient abundance due to cracks or leakage through the cuticular layer and may provide protection from desiccation or UV stress ${ }^{10,13}$. The accessibility of these sites likely explains both how $S$. enterica persists on leaves over long periods of time and why eventually populations decline. This failure to replicate faster than cellular death is most likely because $S$. enterica cannot access the nutrientrich interior of plant tissues on its own.

Recent studies involving $S$. enterica colonization of plants have centered on the identification and characterization of biomultipliers, factors that lead to increased $S$. enterica survival. $S$. enterica exploits changes to the plant environment imparted by other organisms, including infestation with phytophagous insects and infection with bacterial phytopathogens ${ }^{14-22}$. Plants infected with Xanthomonas spp. lead to 
increased persistence of $S$. enterica on tomato (Solanum lycopersicum) leaves ${ }^{17,18}$. Four lineages of $X a n t h o m o n a s$ cause bacterial spot of tomato: $X$. hortorum pv. gardneri (hereafter referred to as $X$. gardneri), $X$. euvesicatoria pv. euvesicatoria, $X$. euvesicatoria pv. perforans, and $X$. vesicatoria ${ }^{23-25}$. These xanthomonads colonize tomato leaf surfaces as epiphytes and enter plant tissue through natural openings to circumvent the epidermis, the first layer of plant defense. Once inside, Xanthomonas colonizes the apoplast where it multiplies and eventually spreads to neighboring leaves, and ultimately other plants. Bacterial spot disease manifests as lesions on leaves, fruit, and stems of tomato plants and ultimately leads to loss of leaves and damaged fruit, resulting in substantial agricultural impact ${ }^{23,24}$.

Although four Xanthomonas lineages cause the same disease on tomato, only a subset of these lineages has a beneficial effect on $S$. enterica populations. In plants colonized by $X$. gardneri, $X$. euvesicatoria pv. euvesicatoria, or, to a lesser extent, $X$. euvesicatoria pv. perforans, $S$. enterica populations on tomato leaves remain steady or even increase over time ${ }^{17}$. Contrastingly, $S$. enterica populations decline in the presence of $X$. vesicatoria or on healthy plants ${ }^{17}$. Core Type III secretion system (T3SS) effectors are shared among the four Xanthomonas species, yet each species also has unique effectors. Of particular interest for this study, $X$. gardneri has a transcription activator-like effector (TALE) AvrHah1 that is not found in $X$. vesicatoria ${ }^{26}$. TALEs induce host transcription by directly binding to the promoter region of target genes using a modular, central repeat domain that recognizes specific host effector binding elements ${ }^{27,28}$. X. gardneri AvrHah1 has over 4,000 potential, predicted binding sites in tomato ${ }^{29}$.

AvrHah1 induces a water soaking phenotype during disease ${ }^{29,30}$, which is characterized by dark green lesions on leaves that reflect an influx of fluid into the apoplast. This water soaking is not observed in $X$. vesicatoria-infected tissues ${ }^{26}$. In tomato, AvrHah1 is required for the induction of two beta helix loop helix (bHLH) transcription factors, bHLH3 and bHLH6 ${ }^{29}$. The spike in transcription for these two regulators leads to induction of at least two additional downstream genes, including a pectate lyase (PL) and pectinesterase $(\mathrm{PE})^{29}$. The current model suggests that $\mathrm{PL}$ alters hygroscopicity in the plant cell wall by releasing oligosaccharides, resulting in an influx of fluid through breaks in the epidermis and the observed water-soaked phenotype ${ }^{29}$. Because the $X$. gardneri avrHah1 mutant reaches the same population level as wildtype $X$. gardneri in planta, it is thought that AvrHah1 is involved in pathogen dispersal or entry into leaf tissue as opposed to acquisition of nutrients during infection ${ }^{29}$.

In this study, we tested the hypothesis that AvrHah1 defines the differential ability between $X$. gardneri and $X$. vesicatoria to enable $S$. enterica persistence in the phyllosphere. We showed that lack of this effector reduced the ability of $X$. gardneri to promote $S$. enterica persistence on tomato leaves. Correspondingly, the addition of avrHah 1 to $X$. vesicatoria allowed this phytopathogen to promote $S$. enterica survival. The data presented here provide evidence that AvrHah1-dependent water soaking may be the mechanism by which the phytopathogenic bacterium benefits the human enteric pathogen.

\section{Results}


AvrHah1 is both necessary and sufficient for X. gardneri-dependent effects on S. enterica persistence

Previously, we had shown that infection with $X$. gardneri promotes survival of $S$. enterica on the leaves of tomato plants ${ }^{19}$. To determine the importance of AvrHah1 for $X$. gardneri-dependent effects on $S$. enterica, we examined the effects of co-inoculation with the $X$. gardneri AvrHah1 DNA-binding domain mutant avrHah1 ${ }^{\triangle \mathrm{DBD}} 30$. Bacterial populations were monitored on tomato plants dip-inoculated with $S$. enterica in the presence or absence of wildtype $X$. gardneri or the $X$. gardneri avrHah $1^{\Delta \mathrm{DBD}}$ mutant. The presence of wildtype $X$. gardneri led to approximately ten-fold higher $S$. enterica populations than the $S$. enterica alone treatment by $6 \mathrm{dpi}$ (Fig. 1a; $\mathrm{P}=0.0058$ ). Conversely, co-inoculation of $S$. enterica with the $X$. gardneri avrHah ${ }^{\triangle \mathrm{DBD}}$ mutant had no significant impact on $S$. enterica (Fig. 1a; $\mathrm{P}=0.889$ ). Xanthomonas populations were monitored over time, and there were no significant differences between treatments (Fig. 1b; P>0.556 for all comparisons).

To determine if avrHah1 is sufficient for Xanthomonas-mediated effects on $S$. enterica persistence, $X$. vesicatoria, which does not impact $S$. enterica persistence, was transformed with a plasmid carrying the avrHah1 gene (pUFR034 (avrHah1)) and tested for its effects on S. enterica. Bacterial populations were determined in tomato plants dip-inoculated with $S$. enterica in the presence or absence of $X$. gardneri, $X$. vesicatoria + pUFR034 (vector alone control), or $X$. vesicatoria + pUFR034 (avrHah1). Co-inoculation of $X$. gardneri or $X$. vesicatoria + pUFR034 (avrHah1) with $S$. enterica resulted in approximately ten-fold higher $S$. enterica populations at $6 \mathrm{dpi}$ than in plants co-inoculated with $X$. vesicatoria + pURF034 and $S$. enterica or inoculated with $S$. enterica alone (Fig. 2a; X. gardneri $\mathrm{P}=6.40 \times 10^{-7}$ and $1.74 \times 10^{-6}$, respectively; $X$. vesicatoria + pUFR034 (avrHah1) $\mathrm{P}=8.17 \times 10^{-5}$ and 0.00019 , respectively). There was no significant difference between the $X$. vesicatoria + pURF034 and $S$. enterica alone treatments $(P=0.8203)$.

Xanthomonas populations were monitored over time, and there were no significant differences between treatments (Fig. 2b; P>0.97). These data demonstrate that the presence of AvrHah1 in Xanthomonas spp. can increase the persistence of $S$. enterica in co-inoculated leaves.

The X. gardneri avrHah1 ${ }^{\triangle \mathrm{DBD}}$ mutant induces transcription of previously identified AvrHah1 targets.

Previous work has shown that AvrHah1 activates expression of multiple tomato genes, including $b H L H 3$, $b H L H 6, P L$, and $P E^{29}$. These targets provide the foundation for the current model of AvrHah1 water soaking. To examine whether these targets play a role in the mechanism by which $X$. gardneri enhances $S$. enterica persistence, we monitored transcription of the genes in leaf samples after inoculation with $S$. enterica in the presence or absence of wildtype $X$. gardneri or the $X$. gardneri avrHah $7^{\triangle \mathrm{DBD}}$ mutant. Under our experimental conditions, plants infected with the $X$. gardneri avrHah ${ }^{\triangle \mathrm{DBD}}$ mutant had the same or higher levels of the four targets compared to plants infected with wildtype $X$. gardneri at 1,3, and $6 \mathrm{dpi}$ (Table 1). In contrast, published results show that wildtype $X$. gardneri Xg153 significantly induces transcription of these genes ( 90-1,300-fold) compared to a corresponding avrHah $1^{\triangle \mathrm{DBD}}$ mutant when infiltrated into tomato Heinz 1706 leaves for 48 hours $^{29}$. To determine the effect of inoculation method (dip-inoculation vs infiltration) on our differing results, we infiltrated MoneyMaker tomato leaves with $X$. 
gardneri 444 wildtype and avrHah $7^{\triangle \mathrm{DBD}}$ mutant following published protocols ${ }^{29}$. Leaf samples were collected 48 hours post-infiltration, and plant gene expression was measured using quantitative PCR (qRTPCR). As with the dip-inoculation experiments, there were no significant differences between leaves inoculated with wildtype $X$. gardneri or the $X$. gardneri avrHah ${ }^{\triangle \mathrm{DBD}}$ mutant for $b H L H 3$, PL, or $P E$ (Table 1). Plants inoculated with wildtype $X$. gardneri had $\sim 30$-fold more $b H L H 6$ transcription than plants inoculated with the $X$. gardneri avrHah ${ }^{\triangle \mathrm{DBD}}$ mutant (Table 1). These data indicate that AvrHah1 may target other host genes in the mechanism that leads to increased $S$. enterica persistence in this system.

Table 1

- Transcription of AvrHah1 targets in tomato leaves after infection with the $X$. gardneri avrHah ${ }^{\triangle \mathrm{DBD}}$ mutant.

\begin{tabular}{|c|c|c|c|c|}
\hline \multirow[b]{2}{*}{ Gene } & \multicolumn{3}{|c|}{ Dip-inoculation } & \multirow{2}{*}{$\begin{array}{l}\text { Infiltration } \\
48 \mathrm{~h}\end{array}$} \\
\hline & $1 \mathrm{dpi}^{\mathrm{a}}$ & $3 \mathrm{dpi}$ & $6 \mathrm{dpi}$ & \\
\hline$b H L H 3$ & $3.28 \pm 2.60$ & $1.10 \pm 0.21$ & $1.21 \pm 0.82$ & $1.71 \pm 0.14$ \\
\hline$b H L H 6$ & $1.88 \pm 0.70$ & $0.76 \pm 0.17$ & $1.52 \pm 1.26$ & $0.04 \pm 0.01$ \\
\hline$P L$ & $2.00 \pm 1.42$ & $1.20 \pm 0.39$ & $4.38 \pm 4.75$ & $9.99 \pm 2.17$ \\
\hline$P E$ & $1.63 \pm 1.02$ & $0.85 \pm 0.26$ & $2.49 \pm 2.71$ & $0.90 \pm 0.19$ \\
\hline
\end{tabular}

$X$. gardneri and $X$. vesicatoria elicit different immune responses in tomato leaves, which are further affected by co-inoculation with S. enterica.

To test the hypothesis that infection with $X$. gardneri alters the plant immune response to the benefit of $S$. enterica, plant defense gene expression was monitored over time in tomato leaves inoculated with $S$. enterica, $X$. gardneri, $X$. vesicatoria, or a combination of each xanthomonad with S. enterica. The SAinducible pathogenesis related protein gene pr1a1 and the JA-inducible proteinase inhibitor gene pin 1 were used as established markers ${ }^{31,32}$ to indirectly monitor these two plant defense pathways with qRTPCR.

At $1 \mathrm{dpi}$, there were few differences in pr1a1 expression between treatments (Fig. 3a). The $X$. gardneri with $S$. enterica co-inoculation treatment was significantly different from the negative control (plants treated with water) $(P=0.0018)$, but all other treatments were statistically the same as the negative control at this time point (Fig. 3a). Compared to the negative control, tomatoes that were inoculated with $X$. gardneri, $X$. gardneri and $S$. enterica, or $X$. vesicatoria showed significant increases in pr1a 1 expression by $3 \mathrm{dpi}$ (Fig. 3a; $\mathrm{P}=0.0205,0.0005$, and 0.0032 , respectively). Contrastingly, tomato plants treated with $X$. vesicatoria and $S$. enterica or $S$. enterica alone had no change in pr1a1 expression compared to the water control at $3 \mathrm{dpi}$ (Fig. 3a; $\mathrm{P}=0.901$ and 0.889 , respectively). By $6 \mathrm{dpi}$, pr1a 1 levels had increased in 
plants inoculated with $X$. gardneri, $X$. gardneri and $S$. enterica, or $X$. vesicatoria with changes reaching approximately 100-10,000-fold compared to water controls (Fig. 3a; $P=5 \times 10^{-7},<1 \times 10^{-7}$, and $7.46 \times 10^{-5}$, respectively). Plants treated with $X$. vesicatoria and $S$. enterica or $S$. enterica alone had no change in pr1a1 expression compared to the water control at 6 dpi (Fig. 3a; $P=0.244$ and 1.00, respectively). Plants inoculated with $X$. vesicatoria and $S$. enterica had pr1a1 levels that were statistically the same as both the water control and the $X$. vesicatoria alone treatment (Fig. 3a; $\mathrm{P}=0.244$ and 0.084 , respectively).

At $1 \mathrm{dpi}$, pin 1 expression levels showed similar trends as pr1a1. There were few differences from the water control except for the $X$. vesicatoria with $S$. enterica treatment which showed 10 -fold higher levels of pin 1 expression (Fig. $3 \mathrm{~b} ; \mathrm{P}<1 \times 10^{-7}$ ). By 3 dpi, treatment with $X$. gardneri led to reduced levels of pin 1 while treatment with $X$. vesicatoria and $S$. enterica resulted in increased pin 1 expression compared to the water control (Fig. 3b; $P=0.0011$ and 0.00097 , respectively). Although inoculation with $X$. vesicatoria or $S$. enterica alone had no change in pin 1 expression compared to the water-inoculated plants at $6 \mathrm{dpi}$ (Fig. 3b; $\mathrm{P}=0.071$ and 0.939 , respectively), inoculation with $X$. gardneri either with or without $S$. enterica led to a 1-3 log decrease in pin 1 levels (Fig. $3 \mathrm{~b} ; \mathrm{P}<1 \times 10^{-7}$ and $3 \times 10^{-7}$, respectively). Co-inoculation with $X$. vesicatoria and $S$. enterica led to a 10 -fold increase in pin 1 expression at 6 dpi (Fig. 3b, $P=0.00048$ ). Taken together, these data show that the two Xanthomonas spp. induce different plant immune responses.

To determine if there was a connection between AvrHah1 and the observed induction of pr1a1 transcription, we monitored pr1a 1 levels at $6 \mathrm{dpi}$ in the $X$. gardneri avrHah $1^{\triangle \mathrm{DBD}}$ mutant. Wildtype $X$. gardneri infection led to increased pr1a 1expression (Fig. $3 c ; \mathrm{P}=1 \times 10^{-7}$ and $5 \times 10^{-7}$ ). Inoculation with the $X$. gardneri avrHah $1^{\triangle \mathrm{DBD}}$ mutant also induced pr1a1 expression compared to the water control (Fig. $3 \mathrm{C} ; \mathrm{P}<$ $\left.1 \times 10^{-7}\right)$. Further, we measured both free and conjugated forms of SA in leaf tissue at 1 and $3 \mathrm{dpi}$ after dip-inoculation with water, $S$. enterica, $X$. gardneri, $X$. gardneri avrHah ${ }^{\triangle \mathrm{DBD}}, X$. vesicatoria, or $S$. enterica with each xanthomonad. SA levels between the treatments were not significantly different ( $P>0.05$; Fig. $\mathrm{S} 1)$. These data demonstrate that avrHah1 is not required for the induction of pr1a1 during $X$. gardneri infection.

To examine the impact of AvrHah1 on plant gene expression in response to $X$. vesicatoria, leaf samples were taken at $6 \mathrm{dpi}$ and examined for pr1a 1 expression. Compared to the negative control, tomatoes that were inoculated with $X$. gardneri, $X$. gardneri and S. enterica, X. vesicatoria + pURF034, X. vesicatoria + pUFR034 (avrHah1), X. vesicatoria + pUFR034 (avrHah1) and S. enterica showed significant increases in pr1a1 expression by $6 \mathrm{dpi}$ (Fig. $3 \mathrm{~d} ; \mathrm{P}=1 \times 10^{-7}, 8 \times 10^{-7}, 0.00048,1.1 \times 10^{-5}$, and $7 \times 10^{-7}$, respectively). Contrastingly, tomato plants treated with $X$. vesicatoria + pURF034 and S. enterica or $S$. enterica alone had no change in pr1a1 expression compared to the water control at 6 dpi (Fig. 3d; $P=0.165$ and 0.174, respectively). Thus, addition of avrHah 1 to $X$. vesicatoria alters the immune response to resemble the host response to $X$. gardneri.

AvrHah1 is not required for electrolyte leakage in X. gardneri-infected tomato leaves. 
Previous work has demonstrated that $X$. gardneri infection leads to more cellular damage in tomato leaves, as measured through electrolyte leakage, than $X$. vesicatoria infection ${ }^{17}$. From those data, it was hypothesized that this increase in cellular damage led to a resulting increase in $S$. enterica persistence in the phyllosphere ${ }^{17}$. Separately, it was shown that AvrHah1 is required for induction of electrolyte leakage in pepper leaves that have been infiltrated with $X$. gardneri ${ }^{30}$. To determine if AvrHah1 is linked to cellular damage and the resulting increases in $S$. enterica populations in tomato, plants were dip-inoculated with water, $S$. enterica, $X$. gardneri, $X$. gardneri avrHah ${ }^{\triangle \mathrm{DBD}}, X$. vesicatoria, or $S$. enterica with each xanthomonad. Leaf samples were collected at 6 dpi. Plants treated with wildtype $X$. gardneri and $S$. enterica or $X$. vesicatoria with or without $S$. enterica had higher levels of electrolyte leakage than the water or $S$. enterica controls ( $P<0.01$, Fig. S2). The $X$. gardneri avrHah $1^{\triangle \mathrm{DBD}}$ treatment resulted in intermediate electrolyte leakage levels that were statistically similar to the water and $S$. enterica controls and both the wildtype $X$. gardneri and $X$. vesicatoria treatments ( $P>0.01$; Fig. S2). The $X$. gardneri avrHah $1^{\triangle \mathrm{DBD}}$ mutant and $S$. enterica treatment gave similar results as the avrHah $1^{\triangle \mathrm{DBD}}$ mutant treatment except that it had statistically lower levels of electrolyte leakage than the two $X$. vesicatoria treatments $(P<0.01 ;$ Fig. S2). These results indicate that specific Xanthomonads cause different levels of electrolyte leakage, but these differences do not correlate with increased S. enterica persistence.

\section{Discussion}

As foliar pathogens, Xanthomonas species survive on both the leaf surface and in the leaf interior. To succeed on the surface, epiphytic bacteria tolerate varying degrees of stress, including UV exposure, desiccation, and nutrient limitation. By actively moving to the leaf interior, xanthomonads can avoid these stresses and thrive. Two goals of this work were to identify which aspect(s) of the $X$. gardneri disease cycle benefit $S$. enterica and to characterize the mechanism that explains the observed phenotypic differences between $S$. enterica populations on $X$. gardneri- and $X$. vesicatoria-infected plants.

For this study, we hypothesized that one or more modifications to the leaf caused by $X$. gardneri infection benefit other leaf community members and lead to increased $S$. enterica survival. The infection process includes aggregation on the leaf surface, entry into the leaf interior, promotion of a water-soaked apoplast, and suppression of host immunity (Fig. 4). Initial S. enterica attachment to the leaf surface appears to be independent of $X$. gardneri as there are no statistical differences between treatments for early S. enterica populations (Fig. 1a; 1 and 3 dpi). Primary lesions develop approximately three dpi and appear as small, circular lesions just visible on the underside of leaves and become more numerous with a water-soaked appearance at four dpi. Based on the rapid appearance of primary lesions, $X$. gardneri quickly modifies the leaf environment to create the macroscopically visible water-soaked lesions. The prevalence of the primary lesions and water-soaked areas continue to increase through six dpi, the final time point sampled in these experiments, but there is little to no growth in individual lesion size at that time. By six dpi, $X$. gardneri-treated plants have ten-fold more $S$. enterica than plants treated with $S$. enterica alone (Fig. 1a). The connection between the observed differences in $S$. enterica populations with the timing of disease progression suggests that the appearance or increased frequency of the water- 
soaked lesions resulted in increased $S$. enterica survival (Fig. 4). Alternatively, or concurrently, the SAinducible pr1a 1 gene begins to show signs of induction as early as $1 \mathrm{dpi}$ with $X$. gardneri (Fig. 3a). The rapid impact of $X$. gardneri on the plant immune response could manifest as increased $S$. enterica survival six days later. Regardless of the mechanism, it takes less than a week in the $X$. gardneri infection process to significantly affect the persistence of $S$. enterica in this environment.

As $X$. gardneri impacts $S$. enterica in the early stages of disease (Fig. 1), the T3SS effectors that have been linked to the acquisition of nutrients and the suppression or evasion of the plant immune response (for review, see ${ }^{33}$ ) could be factors that affect this process. We chose to focus on AvrHah1 because this effector has been linked to the water soaking phenotype in $X$. gardneri and is absent from the $X$. vesicatoria genome ${ }^{26,30}$. Thus, its specificity in $X$. gardneri made it a potential factor that could explain the different effects of these two species on $S$. enterica survival. As shown in this work, AvrHah1 is both necessary and sufficient for Xanthomonas-dependent increases in S. enterica persistence. The $X$. gardneri avrHah $1^{\triangle \mathrm{DBD}}$ mutant is no longer beneficial towards $S$. enterica survival (Fig. 1), and $X$. vesicatoria carrying a plasmid-borne copy of avrHah1 demonstrates a gain of function for this phenotype (Fig. 2). To characterize the mechanism by which AvrHah1 leads to increased $S$. enterica persistence, we examined downstream host targets of AvrHah1. Following dip-inoculation, we saw no significant changes in gene expression for $b H L H 3, b H L H 6, P L$, or $P E$ (Table 1). This result contrasted with previous work demonstrating that infiltration of wildtype $X$. gardneri induced significantly higher transcription of these four genes compared to tomato plants that were infiltrated with the $X$. gardneri avrHah $7^{\triangle \mathrm{DBD}}$ mutant $^{29}$. The two studies differ in several aspects of experimental design, including $X$. gardneri strain, tomato genotype, and inoculation method (dip-inoculation vs infiltration). We found that infiltration of wildtype and mutant $X$. gardneri mildly induced expression of one of the four genes, $b H L H 6$, but not the other three genes (Table 1). These results further support the idea that bacterial strain or tomato genotype likely impact the observed differences in expression between experiments $\left(\right.$ Table $1 ;{ }^{29}$ ). Regardless, these data demonstrate that, in this system, AvrHah1 has different targets that may influence the leaf environment. Schwartz et al identified 4,106 potential AvrHah1 binding sites in tomato ${ }^{29}$. Future characterization of these targets could provide a more detailed mechanism for AvrHah1-mediated increases in S. enterica persistence.

Pathogens, such as $X$. gardneri, have restricted access to the leaf interior through natural openings such as stomates, hydathodes, and wounds. Entry through stomates brings the bacteria to the apoplast, an airfilled, intercellular space ${ }^{34,35}$. During infection with wildtype $X$. gardneri, the apoplast is inundated with host cellular constituents, creating the water-soaked lesions seen in infected tomato plants (Fig. $4 ;{ }^{30}$ ). The water soaking phenotype is absent in plants infected with the $X$. gardneri avrHah ${ }^{1 \mathrm{DBD}}$ mutant. A water-soaked apoplast leads to higher Xanthomonas populations, spread of pathogens into host tissues, suppression of host defense responses, and potentially promotes availability of nutrients ${ }^{36}$. As part of the defense response, plants cause local desiccation to limit pathogen replication (reviewed in ${ }^{37}$ ), and the AvrHah1-dependent water soaking may benefit $S$. enterica by reducing desiccation stress in this niche. In Arabidopsis thaliana, virulent $P$. syringae pv tomato DC3000 causes water potentials that promote 
pathogen growth while avirulent DC3000 are associated with higher levels of desiccation that inhibit bacterial growth ${ }^{38,39}$. In that study, desiccation was measured using a reporter fusion to the proU promoter, which was induced under low water potentials. Utilizing a reporter to monitor water potentials in future experiments could prove to be informative regarding the role of AvrHah1-mediated water soaking in resulting water stress levels.

In addition to desiccation, epiphytic bacteria also experience nutrient limitation on the leaf surface due to the cuticle that restricts diffusion of nutrients from inside the leaf ${ }^{40}$. Migration to the leaf interior may provide access to the many nutrients (sugars, amino acids, etc.) that are transported through the apoplast and into phloem tissue. Although carbon sources such as glucose, fructose, GABA, succinate, and others accumulate in the apoplast, they are typically sequestered in forms that are bound in the cell wall or within cellular vacuoles ${ }^{41}$. Thus, we hypothesized that $X$. gardneri water soaking could release cellular constituents, nutrients, and electrolytes in an accessible form due to changes in host cell membrane permeability (Fig. 4). This result would not be the first example where $S$. enterica utilizes host-derived molecules made available by other bacteria. Plant cell wall breakdown by the soft-rotting pathogen Pectobacterium carotovorum produces oligosaccharides that are thought to be scavenged by $S$. enterica $^{16,21}$. There are also several examples of phytopathogens altering host cell membrane permeability for growth. Pantoea stewartii subsp. stewartii causes water-soaked lesions on corn leaves by damaging cell membranes to release water and nutrients ${ }^{42}$. Water soaking caused by the endophytic pathogen $P$. syringae pv. phaseolicola releases $\mathrm{Ca}^{2+}, \mathrm{Fe}^{2 / 3+}$, and $\mathrm{Mg}^{2+}$ into the apoplast of bean leaves ${ }^{43}$. Our conductance data suggests that electrolyte leakage does not correlate with increased $S$. enterica persistence. Plants co-inoculated with either $X$. gardneri or $X$. vesicatoria and $S$. enterica have increased electrolyte leakage compared to the water and $S$. enterica controls (Fig. S2). However, unlike $X$. gardneri, $X$. vesicatoria infection has no impact on S. enterica populations (Fig. 2). Thus, gross changes in electrolyte leakage cannot explain the $X$-gardneri specific effects on $S$. enterica survival. Although overall differences in electrolyte leakage do not appear to be correlated with $X$. gardneri-mediated increases in $S$. enterica survival, these experiments could have missed changes in the levels of specific electrolytes. Fluctuations in ions of low abundance, like iron, wouldn't have been detected using this approach. Iron has repeatedly been identified as a host-limited nutrient, and pathogens, including $S$. enterica, have multiple mechanisms to scavenge iron from their environment ${ }^{44-46}$. An increase in iron availability could indicate that host manipulations by $X$. gardneri release this critical factor for $S$. enterica growth. Future experiments examining the release of specific ions could elucidate more details of this process.

Invading microorganisms face a number of plant defense responses (antimicrobials, reactive oxygen species (ROS), 'pathogenesis related' (PR) proteins, etc.) that must be overcome to survive in intercellular spaces. The differential ability of $S$. enterica to colonize a range of tomato cultivars suggests an active plant response to the enteric pathogen ${ }^{7}$. Leaf discs treated with the $S$. enterica flagellar epitope flg22 showed increased levels of $\operatorname{ROS}^{47}$, and dip-inoculation of tomatoes with $S$. enterica resulted in a transient induction of the SA-inducible pr1a1 defense gene ${ }^{14}$. Other work has demonstrated that the presence of a virulent pathogen can protect another non-pathogenic epiphyte from host immunity. A nonpathogenic $X$. 
euvesicatoria T3SS mutant regains the ability to grow in planta if co-inoculated with pathogenic $X$. euvesicatoria ${ }^{48}$. The authors showed that the pathogenic $X$. euvesicatoria suppressed host immunity, prevented recognition of LPS from both bacterial strains, and inhibited host defense responses ${ }^{48}$. Further, immune suppression has been linked to water soaking and T3SS in other phytopathogens. $P$. syringae pv tomato induces water soaking and suppresses SA induction using two T3SS effectors HopM1 and $\mathrm{AvrE}^{49,50}$. We hypothesized that $X$. gardneri may manipulate the immune system, performing an analogous function for $S$. enterica here. Co-inoculation of $S$. enterica with $X$. gardneri resulted in sustained induction of pr1a1 and repression of the JA-inducible pin 1 (Fig. 3). Contrastingly, coinoculation of $S$. enterica with $X$. vesicatoria showed no changes in pr1a1 transcription and induced expression of pin 1 (Fig. 3). Despite these differences in pr1a1 gene expression between treatments, there was no effect on levels of free or conjugated SA at one and three dpi for any treatment (Fig. S1). Induction of the SA pathway typically results in a spike in hormone levels followed by a rapid return to basal levels ${ }^{51}$. Thus, it is possible that we missed a transient increase in SA that could have led to pr1a1 induction in the $X$. gardneri-treated plants. Even a short burst of SA production could have beneficial effects on $S$. enterica. Although SA is normally considered to be a mediator of defense responses against bacteria, increased SA levels can result in increased $S$. enterica antibiotic resistance ${ }^{52}$ which could enhance $S$. enterica resistance to stresses in this niche. Alternatively, SA levels may remain constant, and pr1a1 may be induced by a different signaling pathway, such as ethylene. However, because the $X$. gardneri avrHah ${ }^{\triangle \mathrm{DBD}}$ mutant induced pr1a1 expression to the same extent as wildtype $X$. gardneri, we concluded that pr1a1 induction does not contribute to the role of AvrHah1 in increasing S. enterica persistence. Although AvrHah1 is not involved, the importance of SA and other plant hormones in $S$. enterica survival requires further study as there were still significant differences between $X$. gardneri and $X$. vesicatoria in these initial experiments.

In summary, this work identifies one $X$. gardneri factor, AvrHah1, which is both necessary and sufficient for providing conditions that lead to increased $S$. enterica persistence. Although the mechanism by which AvrHah1 promotes $S$. enterica survival remains unclear, several potential explanations have been described and will require further exploration. $X$. gardneri could provide increased nutrient availability through water soaking and/or defense against the plant immune response. Interestingly, $X$. perforans strains carrying the avrHah1 gene have recently been identified in the southeastern United States, and these strains have gained the ability to cause water soaking in pepper leaves ${ }^{53}$. While $X$. perforans is typically found in the southeastern United States, $X$. gardneri is commonly isolated in the midwestern United States. The recent emergence of another species, with different geographic distribution, carrying avrHah1 suggests selective pressure for horizontal transfer of the avrHah1-containing extrachromosomal plasmid between species. Acquisition of avrHah1 may promote Xanthomonas disease and raises the concern that this genetic transfer could lead to an increased risk of $S$. enterica contamination of crops around the country. Continued research in this area will provide fundamental knowledge of how an inhospitable environment changes during infection, resulting in survival of human pathogens and an increased risk of human disease. 


\section{Materials And Methods}

\section{Bacterial strains, media, and culture conditions}

A kanamycin-resistant strain of $S$. enterica serovar Typhimurium 14028s and nalidixic acid strains of $X$. vesicatoria 1111 (ATCC 35937), and X. gardneri 444 (spontaneous Nal-resistant strain; this study) were used as wildtype strains in this study. $S$. enterica was made kanamycin resistant by inserting the aadA gene at the attTn 7 site. The $X$. gardneri avrHah ${ }^{D D B D}$ mutant was obtained from J. Jones ${ }^{30}$ and the $X$. vesicatoria pUFR034 and pUFR034 (avrHah1) strains were made by transforming $X$. vesicatoria 1111 with the Kanamycin-resistant plasmids pUFR034 or pURF034 (avrHah1) ( ${ }^{54}$ and Minsavage, G., unpublished). Bacterial cultures were grown in lysogeny broth (LB) for $S$. enterica at $37^{\circ} \mathrm{C}$ or nutrient broth (NB) for Xanthomonas spp. at $28^{\circ} \mathrm{C}$ with shaking at $200 \mathrm{rpm}$. The antibiotics nalidixic acid (Nal) and Kanamycin (Kan) were used at concentrations of 20 and $50 \mathrm{\mu g} \mathrm{ml}^{-1}$, respectively. Strains used in this study are shown in Table 2.

Table 2 - List of Strains and Primers 


\begin{tabular}{|c|c|c|c|c|}
\hline $\begin{array}{l}\text { Strain } \\
\text { Designation }\end{array}$ & \multicolumn{2}{|l|}{ Genotype } & \multicolumn{2}{|c|}{ Reference or source } \\
\hline JDB1022 & \multicolumn{2}{|c|}{$\begin{array}{l}\text { S. enterica serovar Typhimurium } 14028 \mathrm{~s} ; \mathrm{Kan}^{\mathrm{R}} \text { at } \\
\text { the atfTn } 7 \text { site }\end{array}$} & \multicolumn{2}{|l|}{ This study } \\
\hline JDB1052 & \multicolumn{2}{|l|}{ X. vesicatoria 1111} & \multicolumn{2}{|l|}{ ATCC 35937} \\
\hline JDB1470 & \multicolumn{2}{|l|}{ X. hortorum pv. gardneri 444; $\mathrm{Nal}^{\mathrm{R}}$} & \multicolumn{2}{|l|}{ This study } \\
\hline JDB1459 & \multicolumn{2}{|c|}{$\begin{array}{l}\text { X. hortorum pv. gardneri } 444 \text { avrHah } 1^{D D B D} \text { mutant } \\
\text { (M28) }\end{array}$} & \multicolumn{2}{|l|}{30} \\
\hline JDB1481 & \multicolumn{2}{|c|}{$X$. vesicatoria $1111+$ pUFR034; Kan ${ }^{\mathrm{R}}$} & \multicolumn{2}{|l|}{ This study } \\
\hline JDB1482 & \multicolumn{2}{|c|}{ X. vesicatoria $1111+$ pUFR034 (avrHah1); $\mathrm{Kan}^{\mathrm{R}}$} & \multicolumn{2}{|l|}{ This study } \\
\hline & \multicolumn{4}{|l|}{ Primer sequence $\left(5^{\prime}\right.$ to $\left.3^{\prime}\right)$} \\
\hline Primer & Forward & Reverse & & \\
\hline \multicolumn{4}{|c|}{ Quantitative PCR analysis } & \% \\
\hline $\operatorname{act} 41$ & GCTCTTGACTATGAACAGGAAC & \multicolumn{2}{|c|}{ AAGGACCTCAGGACACCG } & 104 \\
\hline ubi3 & GCCGACTACAACATCCAGAAGG & \multicolumn{2}{|c|}{ TGCAACACAGCGAGCTTAACC } & 105 \\
\hline $\operatorname{pin} 1$ & GCTAAGGAAATAATTGAGAAGGA & \multicolumn{2}{|c|}{ TAAGTCACCACAGGCATT } & 102 \\
\hline pr1a1 & TCAAAGAGCTGATGACTGTG & \multicolumn{2}{|c|}{ GTACCATTGCTTCTCATCGT } & 102 \\
\hline$b H L H 3$ & TGAGAAACAGGGTGATAATGGG & \multicolumn{2}{|c|}{ GTACCCTGTTGGTGCTTCTT } & 109 \\
\hline$b H L H 6$ & TGCAAGAGCTTTCTGTCAATATG & \multicolumn{2}{|c|}{ TAGAGCAGAGGGAGGAAGAA } & 102 \\
\hline$P L$ & TGATTGTGCAATTGGCTTTGG & \multicolumn{2}{|c|}{ TGTTCCTGGCTTTGGATTTACT } & 103 \\
\hline$P E$ & ACAATCTCCCAAGCACAAGG & \multicolumn{2}{|c|}{ GCATATGGGAGAAGGGTGTTTAG } & 97 \\
\hline
\end{tabular}

\section{Plant inoculation}

Tomato cultivar MoneyMaker seeds were purchased commercially (Eden Brothers). No plant material was collected. This study complies with the relevant institutional, national, and international guidelines and legislation for experimental research on plants. Seedlings were cultivated in Professional Growing Mix (Sunshine Redi-earth) with a $16 \mathrm{~h}$ photoperiod at $24^{\circ} \mathrm{C}$ for five weeks. For colonization assays, Xanthomonas bacterial cultures were grown for two days in NB (or NB with Kan for pUFR strains) at $28^{\circ} \mathrm{C}$, and $S$. enterica cultures were grown overnight in $L B$ at $37^{\circ} \mathrm{C}$. Bacterial strains were normalized to an 
optical density at $600 \mathrm{~nm}\left(\mathrm{OD}_{600}\right)$ of 0.2 (for $S$. enterica and $X$. vesicatoria strains), 0.25 (for the $X$. gardneri avrHah $1^{D D B D}$ mutant), and 0.3 (for wildtype $X$. gardneri) in sterile water. These $\mathrm{OD}_{600}$ values correspond to a bacterial population level of $\sim 10^{8} \mathrm{CFU} / \mathrm{ml}$ for the respective strains. Normalized cultures were diluted 1:100 in sterile water for an inoculum level of $\sim 10^{6} \mathrm{CFU} / \mathrm{ml}$. Treatments consisted of individual bacterial strains mixed with equal parts water (S. enterica, $X$. gardneri, $X$. vesicatoria, $X$. gardneri avrHah $1^{D D B D}$ mutant, $X$. vesicatoria pUFR034, or $X$. vesicatoria pUFR034 (avrHah1)), $S$. enterica mixed 1:1 with each individual Xanthomonas strain, or water alone. Prior to inoculation, $0.025 \%$ Sil-Wett was added to water or the bacterial inoculum. Tomato plants were dip-inoculated by inverting plants in either sterile water or the bacterial inoculum for 30 seconds with agitation to prevent bacterial cell settlement. Plants were incubated at high humidity for $48 \mathrm{~h}$ in lidded, plastic bins under grow lights with a $16 \mathrm{~h}$ photoperiod at room temperature $\left(\sim 26^{\circ} \mathrm{C}\right)$. After $48 \mathrm{~h}$, plants were exposed to low humidity conditions (bin lids were removed) during the day and high humidity conditions (bin lids were replaced) during the night. At multiple time points post-inoculation, leaf samples were taken using destructive sampling to determine bacterial populations or collect samples for tomato RNA extraction. For infiltration experiments, MoneyMaker tomato leaves were infiltrated with $X$. gardneri wildtype or the avrHah $7^{\mathrm{DDBD}}$ mutant at $\mathrm{OD}_{600}=0.25$, following published protocols ${ }^{29}$. At $48 \mathrm{~h}$ post-infiltration, two $79 \mathrm{~cm}^{2}$ leaf discs were taken from each of two leaflets on middle leaves and combined for a total of four leaf discs per plant. Samples from four plants per treatment were collected and frozen at $-80^{\circ} \mathrm{C}$ for further processing, as described below.

\section{Bacterial population sampling}

Bacterial populations on leaves were determined as described ${ }^{14}$. Briefly, at indicated times, one $79 \mathrm{~cm}^{2}$ leaf disc was taken from a leaflet on middle leaves using a surface sterilized cork borer ${ }^{18}$. Samples from three plants per treatment per time point were individually homogenized in $500 \mathrm{ml}$ of sterile water in microfuge tubes using a $4.8 \mathrm{~V}$ rotary tool (Dremel, Mt. Prospect, IL) with microcentrifuge tube sample pestle attachment (Fisher Scientific). Homogenates were diluted as needed and spiral plated (Autoplate 4000, Spiral Biotech, Norwood, MA) on LB Kan (for S. enterica) or NB Nal or NB Kan Nal (for Xanthomonas spp.) plates. Resulting colonies were counted after overnight incubation at $37^{\circ} \mathrm{C}$ (for $S$. enterica) or after incubation for three days at $28^{\circ} \mathrm{C}$ (for Xanthomonas spp.) to determine bacterial populations. Although not shown in the figures, bacterial samples were taken at Day 0 to confirm that all bacterial populations were equivalent at the beginning of each experiment. No statistical differences were noted between treatments for any experimental setup at Day $0(P>0.01)$. Experiments were performed with three biological replicates.

\section{RNA isolation}

Tomato gene expression levels were determined as described ${ }^{14}$. At indicated time points, two $79 \mathrm{~cm}^{2}$ leaf discs were taken from each of two leaflets on middle leaves and combined for a total of four leaf discs per plant. Samples from three plants per treatment per time point were collected and frozen at $-80^{\circ} \mathrm{C}$ for 
further processing. RNA was extracted using the MaxwellÒ RSC Plant RNA kit (Promega). Briefly, four leaf discs were homogenized with a mortar and pestle in the presence of liquid nitrogen. Ground tissue was transferred to a small weigh boat containing $450 \mathrm{ml}$ homogenization solution, mixed with a pipette tip, and transferred to a microcentrifuge tube. Samples were then either stored at $-80^{\circ} \mathrm{C}$ until further processed or immediately processed according to manufacturer's instructions using a MaxwellÒ RSC (Promega). RNA samples were eluted in $50 \mathrm{ml}$ volume of RNase-free water and were quantified by NanoDrop (Thermo Scientific). RNA was isolated from three technical replicates for each of three biological replicates.

\section{CDNA synthesis and real-time PCR}

cDNA synthesis and real-time PCR were performed as described ${ }^{14,55,56}$. Briefly, cDNA was synthesized using the iScript cDNA synthesis kit (Bio-Rad). Real-time PCR primers for pin1, pr1a1, bHLH3, bHLH6, PL, and $P E$ were designed with Beacon Designer software (Premier Biosoft International) avoiding template secondary structure (Table 2). Primer efficiencies (Table 2) were calculated using serial dilutions of MoneyMaker genomic DNA and CFX Manager 3.0 software (Bio-Rad). Reference transcripts and primers were chosen based on published works: act41 and ubi3 ${ }^{57}$. Stable expression between treatments was validated using the Best Keeper program and three independent RNA samples from each treatment per biological replicate. Real-time PCR experiments utilized the CFX96 Real-Time System, and data were analyzed with the CFX Manager 3.0 software (Bio-Rad). The mean $C q$ of each target transcript was normalized by the mean $C q$ of each reference gene using the formula: $2^{(-(C q \text { target- } C q \text { reference) })}$. As previously described ${ }^{57}$, we determined the relative expression ratio (RER) of the target gene by dividing the normalized target RNA by a calibrator consisting of the average of the normalized values of the control samples (expression after water treatment in most experiments; expression after infection with wildtype $X$. gardneri in the AvrHah1 target experiments (Table 1)).

\section{Measurement of free and conjugated SA}

Plants were inoculated with S. enterica, $X$. gardneri, $X$. vesicatoria, X. gardneri avrHah $1^{D D B D}$ mutant, with $S$. enterica mixed 1:1 with each individual Xanthomonas strain, or with water alone, as described above. Leaf tissue samples were collected at multiple time points post-inoculation, weighed, flash frozen in liquid nitrogen, and extracted in $600 \mu \mathrm{l}$ of $\mathrm{H}_{2} \mathrm{O}: 1$-propanol:HCL (1:2:0.005) with 200ng of d4-SA as an internal standard. For total SA, $2 \mu$ l of glacial HCL was added, and the sample boiled for 30 min as in ${ }^{58}$. Both free and total SA were phase partitioned with $1 \mathrm{ml} \mathrm{MeCl}$ and the $\mathrm{MeCl}_{2}: 1$-propanol layer was collected and derivatized with trimethylsilydiazomethane, collected by vapor phase extraction on SuperQ columns, eluted in $\mathrm{MeCl}_{2}$ and analyzed using GC-MS as in ${ }^{59}$.

\section{Electrolyte leakage}

Plants were inoculated with S. enterica, $X$. gardneri, $X$. vesicatoria, X. gardneri avrHah $1^{D D B D}$ mutant, with S. enterica mixed 1:1 with each individual Xanthomonas strain, or with water alone, as described above. 
Leaf tissue samples were collected at $6 \mathrm{dpi}$ for bacterial populations (as described above) and electrolyte leakage measurements. Three leaf discs taken from a leaflet from a middle leaf were pooled from each plant, and changes in electrolyte leakage (conductance) were quantified as previously described ${ }^{15}$.

\section{Statistical Analysis}

All statistical analyses were performed using R software (version 2.14.1; R Development Core Team, R Foundation for Statistical Computing, Vienna, Austria [http://www.R-project.org]) as described ${ }^{60}$. Briefly, three biological replicates were performed for each experiment, and samples taken from one replicate were considered as subsamples. Linear regression analysis was used to determine whether bacterial population results differed between treatments for $S$. enterica and Xanthomonas spp. For real-time PCR analysis, three samples were compared for each treatment at each time point using Tukey's HSD test. Results were considered statistically significant at $P<0.01$.

\section{Declarations}

COMPETING INTERESTS

The authors declare no competing interests.

\section{AUTHOR CONTRIBUTIONS}

$\mathrm{KC}$ and $\mathrm{JB}$ designed the experiments for this work, $\mathrm{KC}$ and $\mathrm{AB}$ performed the experiments, and all authors analyzed and interpreted the data. KC and JB wrote the main manuscript text, and all authors reviewed the manuscript.

\section{ACKNOWLEDGEMENTS}

We would like to thank J. Jones and G. Minsavage for Xanthomonas strains and plasmids. We would also like to acknowledge JB's lab members for helpful discussions.

\section{ACKNOWLEDGEMENTS}

We would like to thank J. Jones and G. Minsavage for Xanthomonas strains and plasmids. We would also like to acknowledge JB's lab members for helpful discussions.

\section{DATA AVAILABILITY}

All data generated or analyzed during this study are included in this published article (and its Supplementary Information files).

\section{References}


1. Bidol, S. A. et al. Multistate outbreaks of Salmonella infections associated with raw tomatoes eaten in restaurants-United States, 2005-2006. MMWR Morb Mortal Wkly Rep 56, 909-911 (2007).

2. Toth, B. et al. Outbreak of Salmonella serotype Javiana infections--Orlando, Florida, June 2002. MMWR Morb Mortal Wkly Rep 51, 683-684 (2002).

3. Corby, R. et al. Outbreaks of Salmonella infections associated with eating Roma tomatoes-United States and Canada, 2004. MMWR Morb Mortal Wkly Rep 54, 325-328 (2005).

4. Cummings, K. et al. A multistate outbreak of Salmonella enterica serotype Baildon associated with domestic raw tomatoes. Emerg Infect Dis 7, 1046-1048, doi:10.3201/eid0706.010625 (2001).

5. Guo, X., van lersel, M. W., Chen, J., Brackett, R. E. \& Beuchat, L. R. Evidence of association of salmonellae with tomato plants grown hydroponically in inoculated nutrient solution. App/ Environ Microbio/ 68, 3639-3643, doi:10.1128/aem.68.7.3639-3643.2002 (2002).

6. Barak, J. D., Liang, A. \& Narm, K. E. Differential attachment to and subsequent contamination of agricultural crops by Salmonella enterica. Appl Environ Microbiol 74, 5568-5570, doi:AEM.01077-08 [pii] 10.1128/AEM.01077-08 (2008).

7. Barak, J. D., Kramer, L. C. \& Hao, L. Y. Colonization of tomato plants by Salmonella enterica is cultivar dependent, and type 1 trichomes are preferred colonization sites. App/ Environ Microbio/ 77, 498504, doi:AEM.01661-10 [pii] 10.1128/AEM.01661-10 (2011).

8. Islam, M. et al. Persistence of Salmonella enterica serovar Typhimurium on lettuce and parsley and in soils on which they were grown in fields treated with contaminated manure composts or irrigation water. Foodborne Pathog Dis 1, 27-35, doi:10.1089/153531404772914437 (2004).

9. Islam, M. et al. Fate of Salmonella enterica serovar Typhimurium on carrots and radishes grown in fields treated with contaminated manure composts or irrigation water. Appl Environ Microbio/ 70, 2497-2502 (2004).

10. Kroupitski, Y. et al. Internalization of Salmonella enterica in leaves is induced by light and involves chemotaxis and penetration through open stomata. Appl Environ Microbio/ 75, 6076-6086, doi:10.1128/AEM.01084-09 (2009).

11. Brandl, M. T. \& Mandrell, R. E. Fitness of Salmonella enterica serovar Thompson in the cilantro phyllosphere. Appl Environ Microbio/ 68, 3614-3621 (2002).

12. Cooley, M. B., Miller, W. G. \& Mandrell, R. E. Colonization of Arabidopsis thaliana with Salmonella enterica and enterohemorrhagic Escherichia coli 0157: $\mathrm{H} 7$ and competition by Enterobacter asburiae. Appl Environ Microbiol 69, 4915-4926 (2003).

13. Wagner, G. J. Secreting glandular trichomes: more than just hairs. Plant Physio/ 96, 675-679 (1991).

14. Cowles, K. N., Groves, R. L. \& Barak, J. D. Leafhopper-induced activation of the jasmonic acid response benefits Salmonella enterica in a flagellum-dependent manner. Front Microbio/ 9, 1987 (2018).

15. Harrod, V. L., Groves, R. L., Maurice, M. A. \& Barak, J. D. Frankliniella occidentalis facilitate Salmonella enterica survival in the phyllosphere. PLoS One 16, e0247325 (2021). 
16. Kwan, G., Charkowski, A. O. \& Barak, J. D. Salmonella enterica suppresses Pectobacterium carotovorum subsp. carotovorum population and soft rot progression by acidifying the microaerophilic environment. MBio 4, e00557-00512, doi:10.1128/mBio.00557-12 (2013).

17. Potnis, N., Colee, J., Jones, J. B. \& Barak, J. D. Plant pathogen-induced water-soaking promotes Salmonella enterica growth on tomato leaves. App/ Environ Microbio/ 81, 8126-8134, doi:10.1128/AEM.01926-15 (2015).

18. Potnis, N. et al. Xanthomonas perforans colonization influences Salmonella enterica in the tomato phyllosphere. App/ Environ Microbio/ 80, 3173-3180, doi:10.1128/AEM.00345-14 (2014).

19. Soto-Arias, J. P., Groves, R. \& Barak, J. D. Interaction of phytophagous insects with Salmonella enterica on plants and enhanced persistence of the pathogen with Macrosteles quadrilineatus infestation or Frankliniella occidentalis feeding. PLoS One 8, e79404, doi:10.1371/journal.pone.0079404 PONE-D-13-24740 [pii] (2013).

20. Soto-Arias, J. P., Groves, R. L. \& Barak, J. D. Transmission and retention of Salmonella enterica by phytophagous hemipteran insects. App/ Environ Microbio/ 80, 5447-5456, doi:AEM.01444-14 [pii] 10.1128/AEM.01444-14 (2014).

21. Wells, J. M. \& Butterfield, J. E. Salmonella contamination associated with bacterial soft rot of fresh fruits and vegetables in the marketplace. Plant Disease 81, 867-872 (1997).

22. Barak, J. D. \& Liang, A. S. Role of soil, crop debris, and a plant pathogen in Salmonella enterica contamination of tomato plants. PLoS One 3, e1657, doi:10.1371/journal.pone.0001657 (2008).

23. Jones, J., Stall, R. \& Bouzar, H. Diversity among xanthomonads pathogenic on pepper and tomato. Annu Rev Phytopathol 36, 41-58 (1998).

24. Timilsina, S. et al. Xanthomonas diversity, virulence and plant-pathogen interactions. Nature Rev Microbiol 18, 415-427 (2020).

25. Osdaghi, E. et al. A centenary for bacterial spot of tomato and pepper. Mol Plant Pathol 22, 1500 (2021).

26. Potnis, N. et al. Comparative genomics reveals diversity among xanthomonads infecting tomato and pepper. BMC Genomics 12, 1-23 (2011).

27. Doyle, E. L., Stoddard, B. L., Voytas, D. F. \& Bogdanove, A. J. TAL effectors: highly adaptable phytobacterial virulence factors and readily engineered DNA-targeting proteins. Trends Cell Bio/ 23, 390-398 (2013).

28. Perez-Quintero, A. L. \& Szurek, B. A decade decoded: spies and hackers in the history of TAL effectors research. Annu Rev Phytopathol 57, 459-481 (2019).

29. Schwartz, A. R., Morbitzer, R., Lahaye, T. \& Staskawicz, B. J. TALE-induced bHLH transcription factors that activate a pectate lyase contribute to water soaking in bacterial spot of tomato. Proc Natl Acad Sci USA 114, E897-E903 (2017).

30. Schornack, S., Minsavage, G. V., Stall, R. E., Jones, J. B. \& Lahaye, T. Characterization of AvrHah1, a novel AvrBs3-like effector from Xanthomonas gardneri with virulence and avirulence activity. New Phytologist 179, 546-556 (2008). 
31. Fowler, J. H. et al. Leucine aminopeptidase regulates defense and wound signaling in tomato downstream of jasmonic acid. Plant Cel/ 21, 1239-1251, doi:10.1105/tpc.108.065029 (2009).

32. Tornero, P., Conejero, V. \& Vera, P. A gene encoding a novel isoform of the PR-1 protein family from tomato is induced upon viroid infection. Mol Gen Genet 243, 47-53 (1994).

33. White, F. F., Potnis, N., Jones, J. B. \& Koebnik, R. The type III effectors of Xanthomonas. Mol Plant Pathol 10, 749-766 (2009).

34. Farvardin, A. et al. The apoplast: a key player in plant survival. Antioxidants 9, 604 (2020).

35. Sattelmacher, B. The apoplast and its significance for plant mineral nutrition. New Phytologist 149, 167-192 (2001).

36. Xin, X.-F. et al. Bacteria establish an aqueous living space in plants crucial for virulence. Nature 539, 524-529 (2016).

37. Beattie, G. A. Water relations in the interaction of foliar bacterial pathogens with plants. Annu Rev Phytopathol 49, 533-555 (2011).

38. Wright, C. A. \& Beattie, G. A. Pseudomonas syringae pv. tomato cells encounter inhibitory levels of water stress during the hypersensitive response of Arabidopsis thaliana. Proc Natl Acad Sci USA 101, 3269-3274 (2004).

39. Wright, C. A. \& Beattie, G. A. Bacterial species specificity in proU osmoinducibility and $n p t / l$ and lacZ expression. J Mol Microbiol Biotechnol 8, 201-208 (2004).

40. Lindow, S. E. \& Brandl, M. T. Microbiology of the phyllosphere. App/ Environ Microbio/ 69, 1875-1883 (2003).

41. Rico, A. \& Preston, G. M. Pseudomonas syringae pv. tomato DC3000 uses constitutive and apoplastinduced nutrient assimilation pathways to catabolize nutrients that are abundant in the tomato apoplast. Mol Plant Microbe Interact 21, 269-282 (2008).

42. Ham, J. H., Majerczak, D. R., Arroyo-Rodriguez, A. S., Mackey, D. M. \& Coplin, D. L. WtsE, an AvrEfamily effector protein from Pantoea stewartii subsp. stewartii, causes disease-associated cell death in corn and requires a chaperone protein for stability. Mol Plant Microbe Interact 19, 1092-1102 (2006).

43. O'Leary, B. M. et al. Early changes in apoplast composition associated with defence and disease in interactions between Phaseolus vulgaris and the halo blight pathogen Pseudomonas syringae pv. phaseolicola. Plant Cell Environ 39, 2172-2184 (2016).

44. Hao, L. Y., Willis, D. K., Andrews-Polymenis, H., McClelland, M. \& Barak, J. D. Requirement of siderophore biosynthesis for plant colonization by Salmonella enterica. Appl Environ Microbiol 78, 4561-4570, doi:AEM.07867-11 [pii] 10.1128/AEM.07867-11 (2012).

45. Skaar, E. P. The battle for iron between bacterial pathogens and their vertebrate hosts. PLoS Pathog 6, e1000949 (2010).

46. Parrow, N. L., Fleming, R. E. \& Minnick, M. F. Sequestration and scavenging of iron in infection. Infect Immun 81, 3503-3514 (2013). 
47. Meng, F., Altier, C. \& Martin, G. B. Salmonella colonization activates the plant immune system and benefits from association with plant pathogenic bacteria. Environ Microbio/ 15, 2418-2430, doi:10.1111/1462-2920.12113 (2013).

48. Keshavarzi, M. et al. Basal defenses induced in pepper by lipopolysaccharides are suppressed by Xanthomonas campestris pv. vesicatoria. Mol Plant Microbe Interact 17, 805-815 (2004).

49. Badel, J. L., Shimizu, R., Oh, H.-S. \& Collmer, A. A Pseudomonas syringae pv. tomato avrE1/hopM1 mutant is severely reduced in growth and lesion formation in tomato. Mol Plant Microbe Interact 19, 99-111 (2006).

50. DebRoy, S., Thilmony, R., Kwack, Y.-B., Nomura, K. \& He, S. Y. A family of conserved bacterial effectors inhibits salicylic acid-mediated basal immunity and promotes disease necrosis in plants. Proc Natl Acad Sci USA 101, 9927-9932 (2004).

51. O'Donnell, P. J., Jones, J. B., Antoine, F. R., Ciardi, J. \& Klee, H. J. Ethylene-dependent salicylic acid regulates an expanded cell death response to a plant pathogen. Plant J 25, 315-323 (2001).

52. Sulavik, M. C., Dazer, M. \& Miller, P. F. The Salmonella typhimurium marlocus: molecular and genetic analyses and assessment of its role in virulence. J Bacteriol 179, 1857-1866 (1997).

53. Newberry, E. et al. Independent evolution with the gene flux originating from multiple Xanthomonas species explains genomic heterogeneity in Xanthomonas perforans. App/ Environ Microbio/ 85, e00885-00819 (2019).

54. DeFeyter, R., Kado, C. I. \& Gabriel, D. W. Small, stable shuttle vectors for use in Xanthomonas. Gene 88, 65-72 (1990).

55. Jahn, C. E., Charkowski, A. O. \& Willis, D. K. Evaluation of isolation methods and RNA integrity for bacterial RNA quantitation. J Microbiol Methods 75, 318-324, doi:S0167-7012(08)00254-6 [pii] 10.1016/j.mimet.2008.07.004 (2008).

56. Cowles, K. N., Willis, D. K., Engel, T. N., Jones, J. B. \& Barak, J. D. Diguanylate cyclases AdrA and STM1987 regulate Salmonella enterica exopolysaccharide production during plant colonization in an environment-dependent manner. Appl Environ Microbio/ 82, 1237-1248, doi:10.1128/AEM.03475-15 (2016).

57. Rotenberg, D., Thompson, T. S., German, T. L. \& Willis, D. K. Methods for effective real-time RT-PCR analysis of virus-induced gene silencing. J Virol Methods 138, 49-59, doi:S0166-0934(06)00272-2 [pii] 10.1016/j.jviromet.2006.07.017 (2006).

58. Peng, Z. et al. Xanthomonas translucens commandeers the host rate-limiting step in ABA biosynthesis for disease susceptibility. Proc Natl Acad Sci USA 116, 20938-20946 (2019).

59. Schmelz, E. A., Engelberth, J., Tumlinson, J. H., Block, A. \& Alborn, H. T. The use of vapor phase extraction in metabolic profiling of phytohormones and other metabolites. Plant J 39, 790-808 (2004).

60. Kwan, G., Pisithkul, T., Amador-Noguez, D. \& Barak, J. De novo amino acid biosynthesis contributes to Salmonella enterica growth in alfalfa seedling exudates. Appl Environ Microbio/ 81, 861-873, doi:AEM.02985-14 [pii] 10.1128/AEM.02985-14 (2015). 


\section{Figures}

\section{Figure 1}

TALE AvrHah1 is required for $\boldsymbol{X}$. gardneri-dependent effects on $S$. enterica persistence. Bacterial populations ( $S$. enterica, a; Xanthomonas $\mathbf{b}$ ) on tomato leaves were monitored following treatment with $S$. enterica (black circles), $X$. gardneri (open green triangles), $X$. gardneri avrHah $1^{\mathrm{DDBD}}$ mutant (open orange diamonds), S. enterica $+X$. gardneri (closed green triangles), or $S$. enterica $+X$. gardneri avrHah $1^{\mathrm{DDBD}}$ mutant (closed orange diamonds). All data points from three independent experiments are presented as $\log \mathrm{CFU} / \mathrm{cm}^{2}$. Lines (black, S. enterica; dashed green, $X$. gardneri, dashed orange, $X$. gardneri avrHah $7^{\mathrm{DDBD}}$ mutant; solid green, $S$. enterica $+X$. gardneri; solid orange, $S$. enterica $+X$. gardneri avrHah $1^{\mathrm{DDBD}}$ mutant) correspond to a linear regression model with $95 \%$ confidence intervals represented by the shaded areas. Letters denote significant differences between treatments for the regression lines $(P<0.01)$. Combining three independent experiments, $\mathrm{n}=9$ plants per treatment per time point.

\section{Figure 2}

AvrHah1 is sufficient for Xanthomonas-dependent increases in S. enterica populations. Bacterial populations ( $S$. enterica, a; Xanthomonas $\mathbf{b}$ ) on tomato leaves were monitored following treatment with $S$. enterica (black circles), $X$. gardneri (open green triangles), $X$. vesicatoria pUFR034 (open blue squares), $X$. vesicatoria pUFR034 (avrHah1) (open cyan squares), $S$. enterica $+X$. gardneri (closed green triangles), $S$. enterica $+X$. vesicatoria pUFR034 (closed blue squares), or S. enterica $+X$. vesicatoria pUFR034 (avrHah1) (closed cyan squares). All data points from three independent experiments are presented as $\log \mathrm{CFU} / \mathrm{cm}^{2}$. Lines (black, S. enterica; dashed green, $X$. gardneri, dashed blue, $X$. vesicatoria pUFR034; dashed cyan, $X$. vesicatoria pUFR034 (avrHah1); solid green, $S$. enterica $+X$. gardneri, solid blue, $S$. enterica $+X$. vesicatoria; solid cyan, $S$. enterica $+X$. vesicatoria pUFR034 (avrHah1)) correspond to a linear regression model with $95 \%$ confidence intervals represented by the shaded areas. Letters denote significant differences between treatments for the regression lines $(P<0.01)$. Combining three independent experiments, $\mathrm{n}=9$ plants per treatment per time point.

\section{Figure 3}

Xanthomonas species differentially impact the plant immune responses. Plant gene expression was quantified at days 1, 3, and 6 dpi with water (purple circles), S. enterica (Se; black circles), X. gardneri (Xg; green, open triangles), $X$. gardneri $+S$. enterica (SeXg; green, closed triangles), $X$. vesicatoria (Xv; blue, 
open squares), $X$. vesicatoria $+S$. enterica (SeXv; blue, closed squares), X. gardneri avrHah $7^{\mathrm{DDBD}}$ mutant (Xg DAvr; open, orange diamonds), S. enterica + X. gardneri avrHah ${ }^{\mathrm{DDBD}}$ mutant (SeXg DAvr; closed, orange diamonds), $X$. vesicatoria pUFR034 (avrHah1) (Xv pAvr; open, cyan squares), or S. enterica $+X$. vesicatoria pUFR034 (avrHah1) (SeXv pAvr; closed, cyan squares). Data for the SA-inducible pr1a1 (a, c, d) and JA-inducible pin 1 (b) are displayed as log-transformed relative expression ratios (RER) using water-treated plants as the calibrator. Each symbol represents transcription levels in one tomato plant, and the dashed lines indicate a two-fold change relative to the water control. Transcription was measured in plants from three biological replicates, sampling from three plants per treatment per timepoint for each replicate. Letters denote significant differences between treatments within a single time point $(P<0.05)$. Combining three independent experiments, $\mathrm{n}=9$ plants per treatment per time point.

\section{Figure 4}

Model for $X$. gardneri enhancement of S. enterica persistence in water-soaked leaf tissue. Depiction of the tomato leaf surface (a) shows the location of bacterial cells (Xanthomonas, orange; $S$. enterica, cyan). $X$. gardneri tends to cluster at stomates (pores in the leaf surface) while $S$. enterica has a more random distribution with some cells at cell junctions (wavy lines), stomates (large ovals), and trichomes (not shown). Cross sections of the leaf $(\mathbf{b}, \mathbf{c})$ indicate entry of Xanthomonas bacterial cells (wildtype $X$. gardneri, $\mathbf{b} ; X$. gardneri avrHah $7^{\mathrm{DDBD}}$ mutant, $\mathbf{c}$ ) into the apoplastic space through stomates and some of the resulting consequences to leaf physiology. Water soaking seen during infection by wildtype $X$. gardneri is depicted by the darker green plant cells and the increased amount of water and/or nutrients (purple) in the apoplast (b). This figure was adapted from "Leaf Surface Structure" and "Leaf Anatomy", by BioRender (2021). Retrieved from https://app.biorender.com/biorender-templates.

\section{Supplementary Files}

This is a list of supplementary files associated with this preprint. Click to download.

- SuppFigures.pdf 